\title{
Practical Considerations for Continuum Models Applied to Surface Electrochemistry
}

\author{
Gauthier, Joseph; Dickens, Colin; Ringe, Stefan; Chan, Karen
}

Published in:

ChemPhysChem

Link to article, DOI:

$10.1002 /$ cphc. 201900536

Publication date:

2019

Document Version

Peer reviewed version

Link back to DTU Orbit

Citation (APA):

Gauthier, J., Dickens, C., Ringe, S., \& Chan, K. (2019). Practical Considerations for Continuum Models Applied to Surface Electrochemistry. ChemPhysChem, 20(22), 3074-3080. https://doi.org/10.1002/cphc.201900536

\section{General rights}

Copyright and moral rights for the publications made accessible in the public portal are retained by the authors and/or other copyright owners and it is a condition of accessing publications that users recognise and abide by the legal requirements associated with these rights.

- Users may download and print one copy of any publication from the public portal for the purpose of private study or research.

- You may not further distribute the material or use it for any profit-making activity or commercial gain

- You may freely distribute the URL identifying the publication in the public portal

If you believe that this document breaches copyright please contact us providing details, and we will remove access to the work immediately and investigate your claim. 


\section{Accepted Article}

Title: Practical Considerations for Continuum Models Applied to Surface Electrochemistry

Authors: Joseph Gauthier, Colin Dickens, Stefan Ringe, and Karen Chan

This manuscript has been accepted after peer review and appears as an Accepted Article online prior to editing, proofing, and formal publication of the final Version of Record (VoR). This work is currently citable by using the Digital Object Identifier (DOI) given below. The VoR will be published online in Early View as soon as possible and may be different to this Accepted Article as a result of editing. Readers should obtain the VoR from the journal website shown below when it is published to ensure accuracy of information. The authors are responsible for the content of this Accepted Article.

To be cited as: ChemPhysChem 10.1002/cphc.201900536

Link to VoR: http://dx.doi.org/10.1002/cphc.201900536 


\title{
Practical Considerations for Continuum Models Applied to Surface Electrochemistry
}

\author{
Joseph A. Gauthier*†‡ Dr. Colin F. Dickens* ${ }^{* \dagger \ddagger}$ \\ Dr. Stefan Ringe ${ }^{\dagger \ddagger} \quad$ Prof. Karen $\mathrm{Chan}^{\S \mathbb{I}}$
}

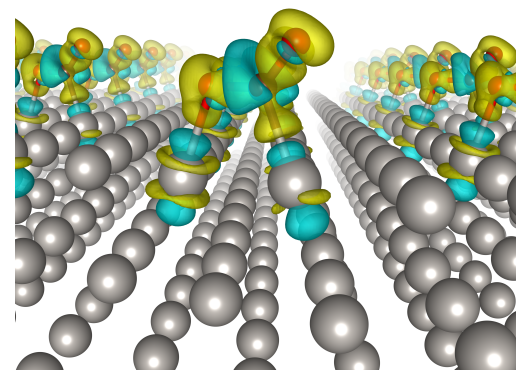

Charge density isosurface illustrating the strong dipole moment exhibited by $\mathrm{CO}_{2}$ on $\mathrm{Pt}$ (111). The yellow surface corresponds to electron density equal to $0.01 \mathrm{e} \AA^{-3}$, while blue corresponds to negative electron density of the same magnitude. The strong dipole moment gives a potential dependence of $\mathrm{CO}_{2}$ adsorption, despite no formal proton/electron transfer process occurring. These potential dependencies can be seen with continuum solvation techniques, which we explore in this work.

\footnotetext{
${ }^{*}$ Contributed equally to this work

${ }^{\dagger}$ SUNCAT Center for Interface Science and Catalysis, Department of Chemical Engineering, Stanford University, Stanford, California 94305, United States

${ }^{\ddagger}$ SUNCAT Center for Interface Science and Catalysis, Department of Chemical Engineering, Stanford University, Stanford, California 94305, United States

$\S$ Department of Physics, Technical University of Denmark, DK-2800, Kgs. Lyngby, Denmark

$\mathbb{I}_{\text {corresponding author }}$
} 


\section{Asbtract}

Modelling the electrolyte at the electrochemical interface remains a major challenge in ab initio simulations of charge transfer processes at surfaces. Recently, the development of hybrid polarizable continuum models/ab initio models have allowed for the treatment of solvation and electrolyte charge in a computationally efficient way. However, challenges remain in its application. Recent literature has reported that large cell heights are required to reach convergence, which presents a serious computational cost. Furthermore, calculations of reaction energetics require costly iterations to tune the surface charge to the desired potential. In this work, we present a simple capacitor model of the interface that illuminates how to circumvent both of these challenges. We derive a correction to the energy for finite cell heights to obtain the large cell energies at no additional computational expense. We furthermore demonstrate that the reaction energetics determined at constant charge are easily mapped to those at constant potential, which eliminates the need to apply iterative schemes to tune the system to a constant potential. These developments together represent more than an order of magnitude reduction of the computational overhead required for the application of polarizable continuum models to surface electrochemistry.

\section{Introduction}

In the past decade, advances in density functional theory (DFT) has paved the way for the theoretical mechanistic study of electrochemical processes. The computational hydrogen electrode (CHE) model [1], in particular, has provided an elegant way to study coupled ion-electron transfer reactions without explicitly modeling charged species. However, the calculation of reaction barriers and the associated kinetics requires the treatment of electrolyte at the interface. To this end, a great deal of effort has been invested to effectively treat charged species at the interface using DFT, ranging from a variety of continuum approximations $[2,3,4,5,6,7,8,9,10,11,12,13,14,15,16,17]$ to a fully ab initio approach.[18, $19,20,21,22]$

Modeling the electrochemical interface with either a continuum electrolyte or with a fully ab initio electrolyte come with their own benefits and challenges.[23] In principle, one of the primary benefits of using a continuum approach is the lack of simulation cell size dependence on the reaction energetics, since the interface can be set to a given potential by modifying the surface charge density. This is not the case in fully ab initio simulations, since the potential changes across the reaction coordinate, proportional to the simulation cell size. $[18,19]$ However, several recent reports on hybrid continuum/ab initio approaches $[24,25,26,27,23]$ have noted that excessively large simulation cells are required to reach convergence, which was attributed to the slow decay of the electrostatic potential in the direction normal to the surface. Furthermore, the iterative scheme to charge the surface to a desired potential can dramatically increase the cost of a calculation. $[28,23]$ In this article, we present a capacitor 
model for the relationship between the reaction energetics and the potential and charge. From this model, we derive a simple correction that relates the energetics determined at small to those at large cells at no additional computational cost. We furthermore demonstrate that the iterative scheme to charge the surface to a given potential is unnecessary, since the energetics at constant charge are trivially mapped to those at constant potential. Combined, these two developments allow for more than an order of magnitude reduction in computational cost required in the determination of electrochemcial reaction energetics with hybrid continuum/ab initio models.

\section{Methods}

This work uses the Vienna ab-initio Software Package[29, 30, 31] in conjunction with VASPsol.[11, 32] Core electrons were modeled with projector augmented wave pseudopotentials, [33] while valence electrons were expanded as planewaves up to a kinetic energy cutoff of $500 \mathrm{eV}$. When optimizing bulk platinum to determine the appropriate lattice constant, the Brillouin zone was sampled with a $12 \times 12 \times 12 \gamma$-centered Monkhorst-Pack[34] k-point mesh. The optimized lattice constant was determined to be 3.990 Å. Exchange and correlation interactions were accounted for using the RPBE functional.[35] A 2x2 supercell of Pt (111) was used as the model system. 4 layers were used in a symmetric unit cell, with the middle two layers fixed and the outer two free to relax.

Geometries were considered optimized when the maximum force on any unconstrained atom in the system was below $0.03 \mathrm{eV} \AA^{-1}$. For each electronic self-consistent field calculation, the density was considered to have converged when the total energy changed by less than $10^{-4} \mathrm{eV}$ between steps.

VASPsol treats the electrolyte at the electrochemical interface as a polarizable continuum. To ensure overall charge neutrality, it places countercharge via the linearized Poisson-Boltzmann (LPB) equation. We chose a Debye length of $3 \AA$, which corresponds to a bulk electrolyte concentration of $1 \mathrm{M}$. Details regarding the implementation of this solvation model can be found in its documentation. [32, 11]

\section{Results and Discussion}

We formulate a simple capacitor model that describes the charging response of surfaces undergoing elementary chemical and electrochemical processes. This simple model shows that, where the capacitance is constant and invariant amongst the states considered, the potential dependence of these elementary processes arises only from the potential of zero charge and capacitance of the surface. We show that the model can be used to correct for the large vacuum required as noted in previous literature, allowing for slab separations comparable to those in vacuum calculations. This correction is essentially due to a potential shift, similar to those derived in previous works. [36, 37] Finally, we apply the model 
to show that the energy difference at constant charge corresponds to the grand canonical free-energy difference at the average potential of the states considered.

\subsection{Formulation of the Model}

In this section we formulate the Taylor expansion of the energy, which expresses it as a function of surface charging properties, the potential of zero charge and the capacitance. We will first consider charging the interface for a given surface state and then consider the energy difference between two states (i.e. representing, for example, reaction or activation energies) under conditions of both constant charge and constant potential.

\subsubsection{For a Single State}

The Taylor expansion of energy as a function of the excess surface charge, $q$, is

$$
E(q)=E(q=0)+\left.q \cdot \frac{\partial E}{\partial q}\right|_{q=0}+\left.\frac{q^{2}}{2} \cdot \frac{\partial^{2} E}{\partial q^{2}}\right|_{q=0}
$$

The first order term is the energy required to bring a unit charge into the slab at zero charge from a specific reference energy level,

$$
\left.\frac{\partial E}{\partial q}\right|_{q=0}=-\mu_{\mathrm{e}, 0}=\Phi_{0}-\Phi_{\mathrm{vac}}
$$

where $\mu_{\mathrm{e}, 0}$ is the Fermi level, which in VASP is calculated relative to the average self-consistent potential. The internal reference level in VASP is different from the bulk electrolyte which is an appropriate electrochemical reference. We therefore express $-\mu_{\mathrm{e}, 0}$ by two terms: the work function at zero charge relative to the bulk electrolyte potential, $\Phi_{0}$, and the shift between the the electrolyte potential and the zero potential reference, $\Phi_{\mathrm{vac}}$, as illustrated in Figure $1 . \Phi_{\mathrm{vac}}$ is printed in VASPsol as the negative of the output flag FERMI_SHIFT and decreases as a function of cell height. Figure 1 also shows that the electrolyte potential is already flat at typical cell sizes, and so the slow convergence of energies with respect to cell height $[24,25,26,27,23]$ arises solely from variations in $\Phi_{\mathrm{vac}}$ rather than a slow decay of countercharge density in the continuum electrolyte.[26]

In a charged LPB system, the absolute value of the potential is fixed even in the presence of periodic boundary conditions due to the additional ionic screening term in the Poisson equation. However, for a finite cell size, the value of the potential in the bulk electrolyte is non-zero and depends on the excess charge, due to the choice of reference potential in VASP. This results in a non-zero boundary integral term to the electrostatic free energy and a constant shift of the potential that corresponds to charge times the potential shift. As we show below, by subtracting out the contribution of $\Phi_{\mathrm{vac}}$, converged energies can be readily obtained from cell heights comparable to those used in vacuum calculations. In principle this reference mismatch arises in any plane-wave DFT code 


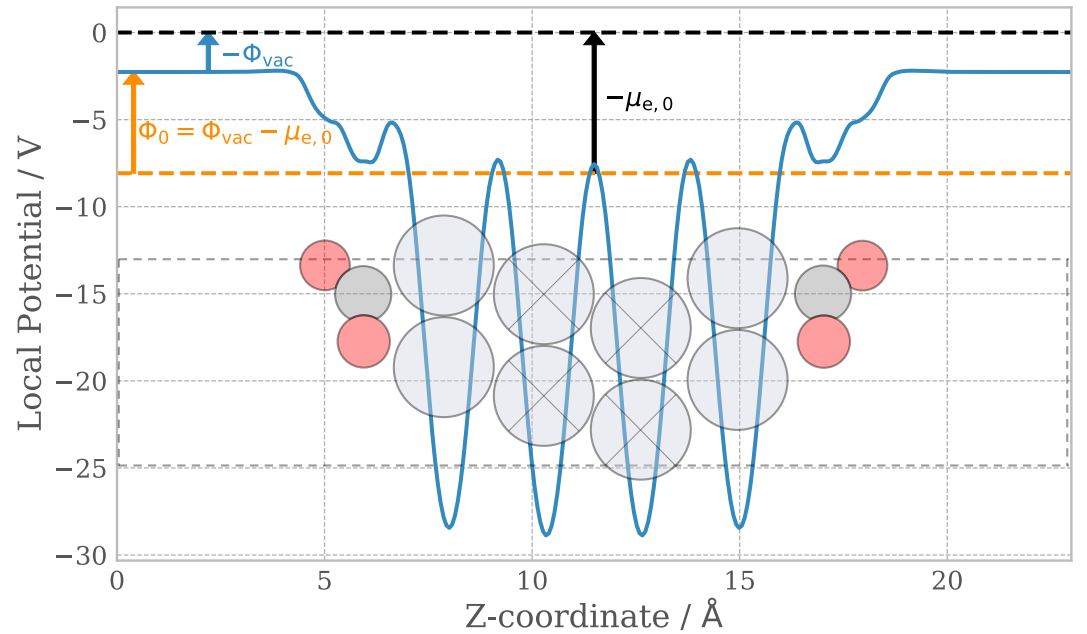

Figure 1: Illustration of the different potential levels in VASPsol using the example of adsorbed $\mathrm{CO}_{2}$ on $\mathrm{Pt}(111)$.

that uses periodic boundary conditions in all three dimensions. For example, in Quantum Espresso's[38] polarizable continuum model Environ[39], an analogous quantity is printed as the shift due to the parabolic periodic boundary condition correction.

The second derivative in the Taylor expansion can be expressed as a function of the capacitance $C$, which we assume to be constant with $q$ :

$$
\left.\frac{\partial^{2} E}{\partial q^{2}}\right|_{q=0}=\left.\frac{\partial \Phi_{0}}{\partial q}\right|_{q=0}=\frac{1}{C}
$$

The energy as a function of surface charge is therefore

$$
E(q)=E(q=0)+q\left(\Phi_{0}-\Phi_{\mathrm{vac}}\right)+\frac{1}{2 C} q^{2} .
$$

This is the expression given by previous works $[40,6,41,42,25]$ with the exception of the nonzero electrolyte level correction, $\Phi_{\text {vac }}$.

Reaction energetics are generally considered as a function of potential. From $q=C\left(\Phi-\Phi_{0}\right)$ and taking the limit $\Phi_{\mathrm{vac}} \rightarrow 0$,

$$
E_{\infty}(\Phi)=E\left(\Phi=\Phi_{0}\right)+\frac{C}{2}\left(\Phi^{2}-\Phi_{0}^{2}\right)
$$

Here the subscript $\infty$ refers to the quantity in the limit of infinite cell height, i.e. in the limit $\Phi_{\mathrm{vac}} \rightarrow 0$. As in previous works, [28, 23] we obtain the grand canonical free-energy of the system by incorporating the exchange of energy from an external electron reservoir: 


$$
\Omega_{\infty}(\Phi)=E(\Phi)-q \Phi=E(\Phi)-C \Phi\left(\Phi-\Phi_{0}\right) .
$$

Here $q$ as before represents the system charge, and $\Phi$ is the system potential (equivalent to the chemical potential of the electron reservoir).

\subsubsection{For the Difference Between Two States}

Under a constant charge $q$ and in the limit of $\Phi_{\text {vac }} \rightarrow 0$, the energy difference between two states are

$$
\begin{aligned}
\Delta E_{\infty}(q) & =E_{2}(q)-E_{1}(q) \\
& =\Delta E(q=0)+\Delta\left(q \Phi_{0}\right)+\frac{\Delta q^{2}}{2 C} \\
& =\Delta E(q=0)+q \Delta \Phi_{0} .
\end{aligned}
$$

Equivalently, we can write the energy difference between these states at constant potential:

$$
\begin{aligned}
\Delta E_{\infty}(\Phi) & =E_{2}(\Phi)-E_{1}(\Phi) \\
& =\Delta E\left(\Phi=\Phi_{0}\right)-\frac{C}{2} \Delta \Phi_{0}^{2} \\
& =\Delta E(q=0)-C \bar{\Phi}_{0} \Delta \Phi_{0} .
\end{aligned}
$$

In the last step, we further introduced the average PZC between the initial and final state, $\bar{\Phi}_{0}$, via the identity $\frac{1}{2} \Delta \Phi_{0}^{2}=\bar{\Phi}_{0} \Delta \Phi_{0}$.

Lastly, we can examine the change in grand canonical free-energy at constant potential and in the limit of $\Phi_{\mathrm{vac}} \rightarrow 0$, between the two states as in (6):

$$
\begin{aligned}
\Delta \Omega_{\infty}(\Phi) & =\Delta E(\Phi)-\Delta(q \Phi) \\
& =\Delta E\left(\Phi=\Phi_{0}\right)+C \Delta \Phi_{0}\left(\Phi-\bar{\Phi}_{0}\right)
\end{aligned}
$$

where we used the capacitor equation in the constant potential limit $\Delta q=$ $-C \Delta \Phi_{0}$.

To illustrate the validity of the model, we use the simple example of $\mathrm{CO}_{2}$ adsorption on $\mathrm{Pt}(111)$. This involves the creation of a strong dipole moment, as is shown in the top panel of Figure 2. Here, the charge density isosurface is calculated as

$$
\rho_{\text {diff }}=\rho_{\text {total }}-\rho_{\text {slab }}-\rho_{\mathrm{CO}_{2}}
$$

The bottom panel shows the agreement between the model and the DFT simulation. We calculate the binding energy of $\mathrm{CO}_{2}$ as

$$
\Delta E_{\mathrm{CO}_{2}}=\frac{1}{2}\left(E_{* \mathrm{CO}_{2}}-E_{*}-2 E_{\mathrm{CO}_{2}(\mathrm{~g})}\right) .
$$

In this equation, $\Delta E_{\mathrm{CO}_{2}}$ is the binding energy of $\mathrm{CO}_{2}, E_{* \mathrm{CO}_{2}}$ is the energy of the system of the slab with two $\mathrm{CO}_{2}$ molecules adsorbed (i.e. due to the 
symmetry in the z-direction, as illustrated in Figure 1) at a given potential, $E_{*}$ is the energy of the clean slab at the same potential, and $E_{\mathrm{CO}_{2}(\mathrm{~g})}$ is the energy of a gas-phase $\mathrm{CO}_{2}$ molecule. In the bottom panel of Figure 2, the points represent the calculated change in grand canonical free-energy associated with $\mathrm{CO}_{2}$ adsorption as obtained from Eq. 6 and Eq. 13 while the line represents the model given in Equation 11. The points have been corrected to achieve the limit $\Phi_{\text {vac }} \rightarrow 0$ (which the model, Eq. 11, assumes), demonstrated in the next section. $\Phi_{0}$ and $E\left(\Phi=\Phi_{0}\right)$ from the model were determined directly from $\mu_{\mathrm{e}, 0}$ and $\Phi_{\text {vac }}$, while $C$ was determined by a linear fit of $q(\Phi)$, illustrated in SI Note 1. We find the capacitance of the clean $\mathrm{Pt}$ (111) surface to be $12.6 \mu \mathrm{F} \mathrm{cm}^{-2}$, and the capacitance with $\mathrm{CO}_{2}$ adsorbed in a $(2 \times 2)$ supercell of $\mathrm{Pt}(111)$ to be $10.1 \mu \mathrm{F} \mathrm{cm}^{-2}$. This small change in capacitance contributes to the slight non-linearity observed in the bottom panel of Figure 2. We note that in larger cell sizes, this change in capacitance decays quite rapidly. In a (4x4) supercell, the capacitance decreases by only $0.1 \mu \mathrm{F} \mathrm{cm}^{-2}$. Since the quadratic term is proportional to the change in capacitance, we therefore find the assumption of constant capacitance between states to be quite robust to the addition of adsorbates. 


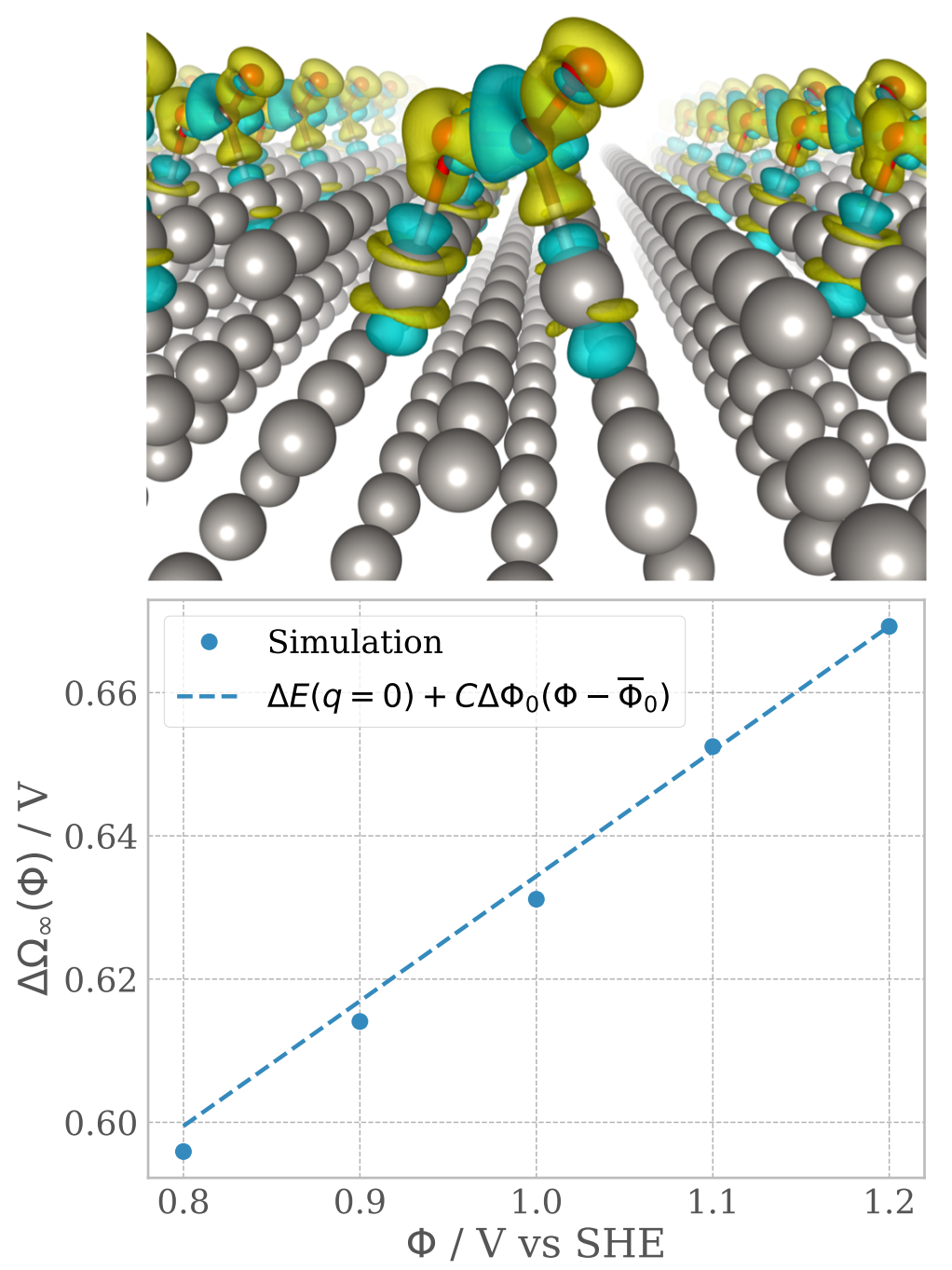

Figure 2: Demonstration of the simple model in describing the electrostatic response of $\mathrm{Pt}$ (111) to the adsorption of $\mathrm{CO}_{2}$. Top: Charge density difference isosurface demonstrating the strong dipole moment created by $\mathrm{CO}_{2}$ at the surface. Yellow corresponds to an isosurface of positive electron density equal to $0.01 \mathrm{e} \AA^{-3}$, while blue corresponds to a negative electron density of $0.01 \mathrm{e}$ $\AA^{-3}$. Bottom: Agreement between the derived model and the associated DFT calculations. Here points illustrate calculated binding energies from DFT, and the dashed line corresponds to the model derived in Eq. 6 . 


\subsection{Correcting for Finite Separation Between Periodic Im- ages}

In what follows, we show the corrections needed to obtain the energy associated with a zero potential reference in bulk electrolyte using finite cell heights.

\subsubsection{Correction at Constant Charge}

We consider again Eq. (9), which was derived in the limit of infinite cell height, i.e. $\Phi_{\mathrm{vac}} \rightarrow 0$. If we neglect to make this assumption and isolate the terms that depend on $\Phi_{\text {vac }}$, we see:

$$
\Delta E(q)=\Delta E(q=0)+q \Delta\left(\Phi_{0}\right)-q \Delta\left(\Phi_{\mathrm{vac}}\right) .
$$

In the limit of infinite separation of the surface slabs, the potential in the electrolyte region $\Phi_{\mathrm{vac}} \rightarrow 0$, and therefore also $\Delta \Phi_{\mathrm{vac}} \rightarrow 0$ and $\mu_{\mathrm{e}, 0} \rightarrow-\Phi_{0}$. We can then write for the extrapolated energy difference at infinite separation of the slabs

$$
\Delta E_{\infty}(q)=\Delta E(q)+q \Delta \Phi_{\mathrm{vac}} .
$$

Here $\Delta E_{\infty}(q)$ is the change in energy between two states at constant charge in the limit of an infinite separation between periodic slabs, $\Delta E(q)$ is the difference in energy with finite separation.

To demonstrate this, we have calculated the binding energy of $\mathrm{CO}_{2}$ on $\mathrm{Pt}$ (111) at a constant surface charge density of $6 \mu \mathrm{C} \mathrm{cm}^{-2}$ at varying amounts of separation between periodic images. Figure 3a shows the agreement between the corrected energy and the large vacuum limit, while Figure $3 \mathrm{~b}$ shows the decay of $\Phi_{\text {vac }}$ with separation distance. 

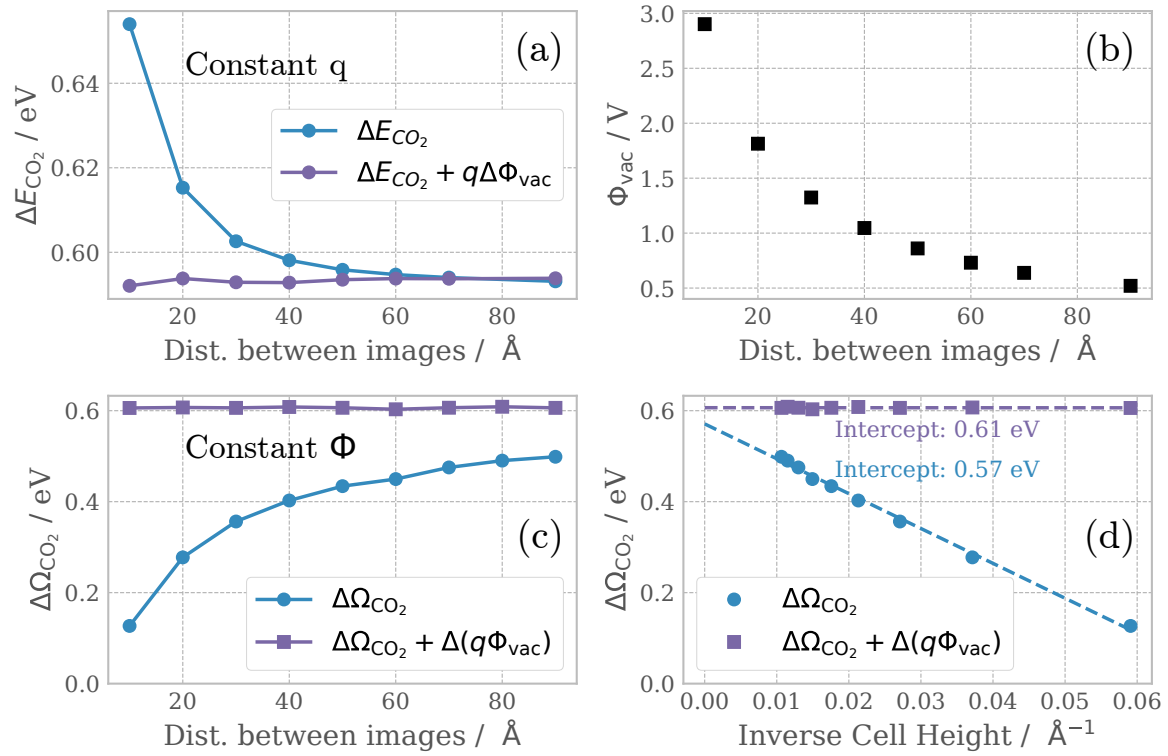

Figure 3: Illustration of correction for finite separation between periodic slabs for a calculation at constant charge and constant potential. (a) The convergence of the corrected and uncorrected energy for large separations between periodic images at a constant surface charge of $6 \mu \mathrm{C} \mathrm{cm}^{-2}$. (b) The decay of the the electrolyte potential, as it goes to zero in the limit of infinite separation between periodic images. (c) The convergence of the corrected and uncorrected constant potential grand canonical free-energy difference for large separations between periodic images. (d) The extrapolation of the energy difference to infinite cell size. 


\subsubsection{Correction at Constant Potential}

Constant potential calculations, which are performed with the initial and final state at different charges such that the resultant potential remains the same, are of particular interest since they reflect experimental conditions. Beginning with (11) without taking the limit $\Phi_{\mathrm{vac}} \rightarrow 0$, we see at constant potential:

$$
\begin{aligned}
\Delta \Omega(\Phi) & =\Delta E(q=0)+C\left(\Delta\left(\Phi_{0} \Phi_{\mathrm{vac}}\right)-\Phi \Delta \Phi_{\mathrm{vac}}\right)+C \Delta \Phi_{0}\left(\Phi-\bar{\Phi}_{0}\right) \\
& =\Delta E(q=0)+C \Delta \Phi_{0}\left(\Phi-\bar{\Phi}_{0}\right)-\Delta\left(\Phi_{\mathrm{vac}} C\left(\Phi-\Phi_{0}\right)\right) \\
& =\Delta E(q=0)+C \Delta \Phi_{0}\left(\Phi-\bar{\Phi}_{0}\right)-\Delta\left(q \Phi_{\mathrm{vac}}\right)
\end{aligned}
$$

Using the same argument as in the case of constant charge, in the limit of infinite separation between periodic images, $\Phi_{\mathrm{vac}} \rightarrow 0$ and so we have:

$$
\Delta \Omega_{\infty}(\Phi)=\Delta \Omega(\Phi)+\Delta\left(q \Phi_{\mathrm{vac}}\right) .
$$

Where, as before, $\Delta \Omega_{\infty}(\Phi)$ refers to the change in grand canonical freeenergy in the limit of infinite separation between periodic images, $\Delta \Omega(\Phi)$ to the finite energy difference, $q$ to the charge, and $\Phi_{\text {vac }}$ to the average electrostatic potential in the calculation. Note that both $q$ and $\Phi_{\text {vac }}$ vary from initial to final state.

Figure 3c illustrates the efficiency of this correction. The correction is generally larger in magnitude for constant potential calculations because the charge is changing with each state. This charge is multiplied by the electrolyte region electrostatic potential in the correction, which can be quite large, 1-2 V, for small cell heights. Whereas in the case of constant charge, there is significant error cancellation because the charge only multiplies the difference in average electrostatic potential. Figure 3d illustrates an extrapolation based method to obtain the infinite cell change in grand canonical free-energy, as illustrated in Ref. numbers[24]. We note that the remaining small differences in intercept are likely due to the tolerance on the applied potential $( \pm 0.02 \mathrm{~V})$ when optimizing the excess surface charge.

Overall, by avoiding the extremely large vacuums reported in literature, calculation times can be substantially reduced. In our example system, this results in a 7-fold speed increase per scf-step between the largest and smallest systems tested. As noted previously, the energetic variation corrected for here is general to polarizable continuum models in plane-wave codes that do not use an open boundary condition normal to the surface. Some codes, for example the polarizable continuum code Environ[39] implemented in QUANTUM-ESPRESSO[38] automatically corrects for the energetic shift due to the nonzero electrolyte potential. However, as noted by the VASPsol Github page[32] which advises users to add this correction to energies, VASPsol does not automatically add this correction, resulting in energetic deviations in a significant portion of literature. 


\subsection{Mapping Energetics at Constant Charge to Constant Potential}

We now demonstrate that, in the limit of $\Phi_{\mathrm{vac}} \rightarrow 0$ and constant capacitance, the change in energy at constant charge corresponds to the change grand canonical free-energy at a constant potential corresponding to the average potential between the states considered.

We recall from Eq. (9), that at constant charge the expression for the change in energy becomes:

$$
\Delta E_{\infty}(q)=\Delta E(q=0)+q \Delta \Phi_{0}
$$

To compare this with (11), we will evaluate at the average potential of the two states, 1 and 2 . This can be done by rearranging:

$$
\begin{aligned}
q_{1}=q_{2} & =C\left(\Phi_{1}-\Phi_{0,1}\right)=C\left(\Phi_{2}-\Phi_{0,2}\right) \\
& =C\left(\bar{\Phi}-\bar{\Phi}_{0}\right) .
\end{aligned}
$$

Here $\Phi_{1}$ and $\Phi_{2}$ refer to the potential in states 1 and 2 respectively, while $\Phi_{0,1}$ and $\Phi_{0,2}$ refer to the potential at zero charge in states 1 and 2 respectively.

Substituting this expression for $q$ into (18), we see:

$$
\Delta E_{\infty}(\bar{\Phi})=\Delta E(q=0)+C \Delta \Phi_{0}\left(\bar{\Phi}-\bar{\Phi}_{0}\right)
$$

Comparing this expression to (10), we see that if the capacitance is constant and $\Phi_{\mathrm{vac}} \rightarrow 0$, the change in energy at constant charge is equal to the change in grand canonical free-energy at the average potential. This result agrees with the results derived via Legendre transformation in Ref. numbers[43], and is a more general result of that in Ref. numbers[44], where it was shown that the transition state at constant charge and potential were identical.

To demonstrate this equivalence, we have calculated the binding energy of $\mathrm{CO}_{2}$ on $\mathrm{Pt}$ (111) at both constant charge and constant potential, shown in Figure 4. 


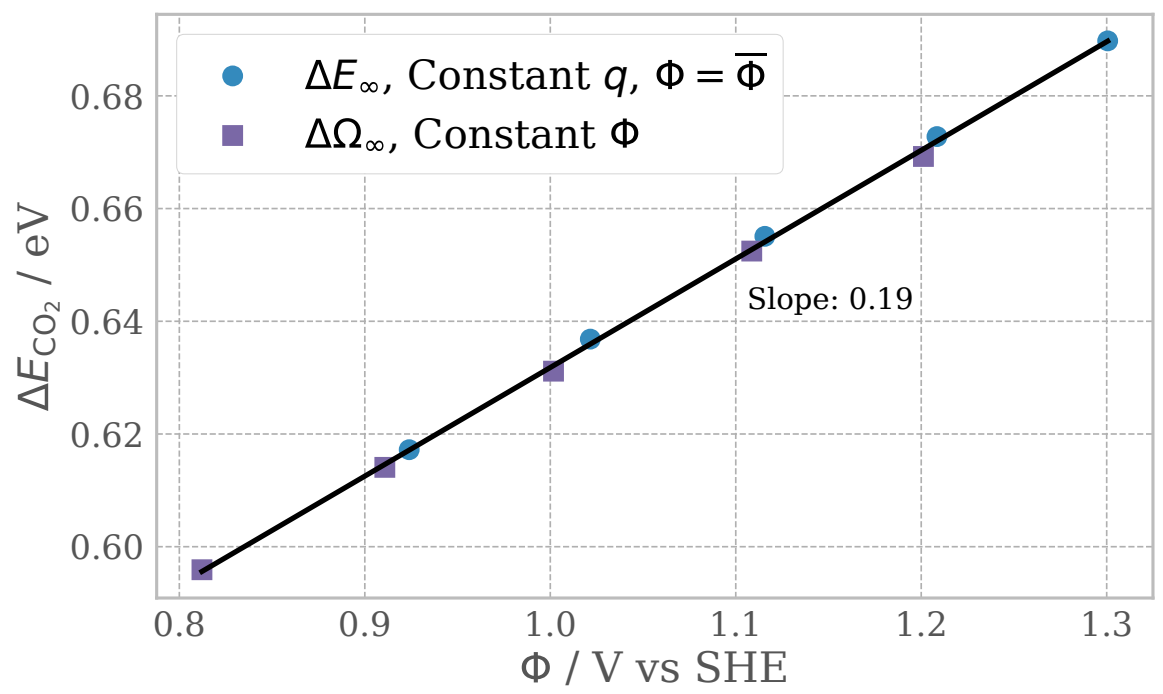

Figure 4: Agreement of the change in binding energy of $\mathrm{CO}_{2}$ on $\mathrm{Pt}(111)$ at constant charge and the change in binding grand canonical free-energy at constant potential.

The agreement between these two quantities represents a further reduction in computational time when applying continuum methods to surface electrochemistry at constant potential. The iterative procedure by which the potential is set to a desired value, achieved by optimizing the system charge, is a significant cost increase compared to a standard calculation, approximately a factor of two to three. [28, 23] Rather than calculate the grand canonical free-energy at a fixed potential, one can instead do the simpler calculation at constant charge. When evaluated at the average potential in conjunction with the corrections for finite cell height, this gives an identical relationship between energy and potential.

\section{Conclusions}

In summary, we have presented a simple model for reaction energetics determined from hybrid continuum/ab initio models of the electrochemcial interface, derived from a Taylor expansion of the energy. With this model, we derived simple corrections that allow for the calculation of energy differences at reasonable separation between periodic images, comparable to those seen in vacuum calculations. We further use the model to illustrate that the grand canonical free-energy for a reaction process at constant potential is exactly equivalent to the energy difference at constant charge, when applied in conjunction with the finite cell height correction. These two developments together give rise to more 
than an order of magnitude reduction in the computational overhead required to apply polarizable continuum models to surface electrochemistry investigations.

This work was supported by the U.S. Department of Energy, Chemical Sciences, Geosciences, and Biosciences (CSGB) Division of the Office of Basic Energy Sciences, via Grant DE-AC02-76SF00515 to the SUNCAT Center for Interface Science and Catalysis. C.F.D. acknowledges fellowship support from the National Science Foundation Graduate Research Fellowship (Grant No. DGE114747). K.C. acknowledges a research grant (9455) from VILLUM FONDEN." Some of the computing for this project was performed on the Sherlock cluster. We would like to thank Stanford University and the Stanford Research Computing Center for providing computational resources and support that contributed to these research results. This research used resources of the National Energy Research Scientific Computing Center (NERSC), a U.S. Department of Energy Office of Science User Facility operated under Contract No. DE-AC02$05 \mathrm{CH} 11231$

\section{References}

[1] Jens Kehlet Nørskov, Jan Rossmeisl, Ashildur Logadottir, LRKJ Lindqvist, John R Kitchin, Thomas Bligaard, and Hannes Jonsson. Origin of the overpotential for oxygen reduction at a fuel-cell cathode. The Journal of Physical Chemistry B, 108(46):17886-17892, 2004.

[2] Ikutaro Hamada, Osamu Sugino, Nicéphore Bonnet, and Minoru Otani. Improved modeling of electrified interfaces using the effective screening medium method. Physical Review B, 88(15):155427, 2013.

[3] Minoru Otani and Osamu Sugino. First-principles calculations of charged surfaces and interfaces: A plane-wave nonrepeated slab approach. Physical Review B, 73(11):115407, 2006.

[4] AY Lozovoi, A Alavi, J Kohanoff, and RM Lynden-Bell. Ab initio simulation of charged slabs at constant chemical potential. The Journal of Chemical Physics, 115(4):1661-1669, 2001.

[5] Christopher D Taylor, Sally A Wasileski, Jean-Sebastien Filhol, and Matthew Neurock. First principles reaction modeling of the electrochemical interface: Consideration and calculation of a tunable surface potential from atomic and electronic structure. Physical Review B, 73(16):165402, 2006.

[6] Mikhail Mamatkulov and Jean-Sébastien Filhol. An ab initio study of electrochemical vs. electromechanical properties: the case of $\mathrm{CO}$ adsorbed on a Pt(111) surface. Physical Chemistry Chemical Physics : PCCP, 13(17):7675-7684, 2011. 
[7] Ya-Hui Fang and Zhi-Pan Liu. Surface phase diagram and oxygen coupling kinetics on flat and stepped pt surfaces under electrochemical potentials. The Journal of Physical Chemistry C, 113(22):9765-9772, 2009.

[8] Ya-Hui Fang and Zhi-Pan Liu. Mechanism and tafel lines of electrooxidation of water to oxygen on ruo2 (110). Journal of the American Chemical Society, 132(51):18214-18222, 2010.

[9] Stefan Ringe, Harald Oberhofer, Christoph Hille, Sebastian Matera, and Karsten Reuter. Function-space-based solution scheme for the size-modified poisson-boltzmann equation in full-potential dft. Journal of chemical theory and computation, 12(8):4052-4066, 2016.

[10] Ryosuke Jinnouchi and Alfred B. Anderson. Aqueous and surface redox potentials from self-consistently determined gibbs energies. Journal of Physical Chemistry C, 112(24):8747-8750, 2008.

[11] Kiran Mathew and Richard G Hennig. Implicit self-consistent description of electrolyte in plane-wave density-functional theory. arXiv preprint arXiv:1601.03346, 2016.

[12] G Fisicaro, L Genovese, O Andreussi, N Marzari, and S Goedecker. A generalized poisson and poisson-boltzmann solver for electrostatic environments. The Journal of Chemical Physics, 144(1):014103, 2016.

[13] Ya-Hui Fang, Guang-Feng Wei, and Zhi-Pan Liu. Theoretical modeling of electrode/electrolyte interface from first-principles periodic continuum solvation method. Catalysis today, 202:98-104, 2013.

[14] Kendra Letchworth-Weaver and TA Arias. Joint density functional theory of the electrode-electrolyte interface: Application to fixed electrode potentials, interfacial capacitances, and potentials of zero charge. Physical Review B, 86(7):075140, 2012.

[15] Nathan Keilbart, Yasuaki Okada, Aion Feehan, Shin'ichi Higai, and Ismaila Dabo. Quantum-continuum simulation of the electrochemical response of pseudocapacitor electrodes under realistic conditions. Physical Review B, 95(11):115423, 2017.

[16] Ravishankar Sundararaman and Kathleen Schwarz. Evaluating continuum solvation models for the electrode-electrolyte interface: Challenges and strategies for improvement. The Journal of Chemical Physics, 146(8):084111, 2017.

[17] Noriyuki Minezawa and Shigeki Kato. Efficient implementation of threedimensional reference interaction site model self-consistent-field method: Application to solvatochromic shift calculations. The Journal of Chemical Physics, 126(5):054511, 2007. 
[18] Karen Chan and Jens K Nørskov. Potential dependence of electrochemical barriers from ab initio calculations. The Journal of Physical Chemistry Letters, 7(9):1686-1690, 2016.

[19] Karen Chan and Jens K Nørskov. Electrochemical barriers made simple. The Journal of Physical Chemistry Letters, 6(14):2663-2668, 2015.

[20] Nicéphore Bonnet, Tetsuya Morishita, Osamu Sugino, and Minoru Otani. First-principles molecular dynamics at a constant electrode potential. Physical Review Letters, 109(26):266101, 2012.

[21] Jan Rossmeisl, Egill Skúlason, Mårten E Björketun, Vladimir Tripkovic, and Jens K Nørskov. Modeling the electrified solid-liquid interface. Chemical Physics Letters, 466(1-3):68-71, 2008.

[22] Martina Lessio, Thomas P Senftle, and Emily A Carter. Hydride shuttle formation and reaction with co2 on gap (110). ChemSusChem, 11(9):15581566, 2018.

[23] Joseph A Gauthier, Stefan Ringe, Colin F Dickens, Alejandro J Garza, Alexis T Bell, Martin Head-Gordon, Jens K Nørskov, and Karen Chan. Challenges in modeling electrochemical reaction energetics with polarizable continuum models. ACS Catalysis, 9:920-931, 2018.

[24] Maxime Van den Bossche, Egill Skúlason, Christoph Rose-Petruck, and Hannes Jónsson. Assessment of constant-potential implicit solvation calculations of electrochemical energy barriers for h2 evolution on pt. The Journal of Physical Chemistry C, 2018.

[25] Stephan N Steinmann, Carine Michel, Renate Schwiedernoch, and Philippe Sautet. Impacts of electrode potentials and solvents on the electroreduction of co 2: a comparison of theoretical approaches. Physical Chemistry Chemical Physics, 17(21):13949-13963, 2015.

[26] Stephan N Steinmann and Philippe Sautet. Assessing a first-principles model of an electrochemical interface by comparison with experiment. The Journal of Physical Chemistry C, 120(10):5619-5623, 2016.

[27] Stephan N Steinmann, Philippe Sautet, and Carine Michel. Solvation free energies for periodic surfaces: comparison of implicit and explicit solvation models. Physical Chemistry Chemical Physics, 18(46):31850-31861, 2016.

[28] Jason D Goodpaster, Alexis T Bell, and Martin Head-Gordon. Identification of possible pathways for $\mathrm{c}-\mathrm{c}$ bond formation during electrochemical reduction of co2: New theoretical insights from an improved electrochemical model. The Journal of Physical Chemistry Letters, 7(8):1471-1477, 2016.

[29] G. Kresse and J. Hafner. Ab initio molecular dynamics for liquid metals. Physical Review B, 47(1):558-561, 1993. 
[30] G. Kresse and J. Furthmüller. Efficient iterative schemes for ab initio total-energy calculations using a plane-wave basis set. Physical Review B, 54(16):11169-11186, 1996.

[31] G. Kresse and J. Furthmüller. Efficiency of ab-initio total energy calculations for metals and semiconductors using a plane-wave basis set. Computational Materials Science, 6(1):15-50, 1996.

[32] Kiran Mathew, Ravishankar Sundararaman, Kendra Letchworth-Weaver, TA Arias, and Richard G Hennig. Implicit solvation model for densityfunctional study of nanocrystal surfaces and reaction pathways. The Journal of Chemical Physics, 140(8):084106, 2014.

[33] G. Kresse. From ultrasoft pseudopotentials to the projector augmentedwave method. Physical Review B, 59(3):1758-1775, 1999.

[34] Hendrik J Monkhorst and James D Pack. Special points for brillouin-zone integrations. Physical Review B, 13(12):5188, 1976.

[35] BHLB Hammer, Lars Bruno Hansen, and Jens Kehlet Nørskov. Improved adsorption energetics within density-functional theory using revised perdew-burke-ernzerhof functionals. Physical Review B, 59(11):7413, 1999.

[36] Lennart Bengtsson. Dipole correction for surface supercell calculations. Physical Review B, 59(19):12301, 1999.

[37] Oliviero Andreussi and Nicola Marzari. Electrostatics of solvated systems in periodic boundary conditions. Physical Review B, 90(24):245101, 2014.

[38] Paolo Giannozzi, Stefano Baroni, Nicola Bonini, Matteo Calandra, Roberto Car, Carlo Cavazzoni, Davide Ceresoli, Guido L Chiarotti, Matteo Cococcioni, Ismaila Dabo, Andrea Dal Corso, Stefano de Gironcoli, Stefano Fabris, Guido Fratesi, Ralph Gebauer, Uwe Gerstmann, Christos Gougoussis, Anton Kokalj, Michele Lazzeri, Layla Martin-Samos, Nicola Marzari, Francesco Mauri, Riccardo Mazzarello, Stefano Paolini, Alfredo Pasquarello, Lorenzo Paulatto, Carlo Sbraccia, Sandro Scandolo, Gabriele Sclauzero, Ari P Seitsonen, Alexander Smogunov, Paolo Umari, and Renata M Wentzcovitch. QUANTUM ESPRESSO: A modular and opensource software project for quantum simulations of materials. Journal of Physics: Condensed Matter, 21(39):395502, sep 2009.

[39] Oliviero Andreussi, Ismaila Dabo, and Nicola Marzari. Revised selfconsistent continuum solvation in electronic-structure calculations. The Journal of Chemical Physics, 136(6):064102, 2012.

[40] Ismaila Dabo, Yanli Li, Nicéphore Bonnet, and Nicola Marzari. Ab initio electrochemical properties of electrode surfaces. Fuel Cell Science: Theory, Fundamentals, and Biocatalysis, pages 415-431, 2010. 
[41] Nicéphore Bonnet, Ismaila Dabo, and Nicola Marzari. Chemisorbed molecules under potential bias: detailed insights from first-principles vibrational spectroscopies. Electrochimica Acta, 121:210-214, 2014.

[42] Jean-Sébastien Filhol and Marie-Liesse Doublet. An ab initio study of surface electrochemical disproportionation: The case of a water monolayer adsorbed on a pd ( $\left.\begin{array}{lll}1 & 1 & 1\end{array}\right)$ surface. Catalysis today, 202:87-97, 2013.

[43] Nicolas G Hörmann, Oliviero Andreussi, and Nicola Marzari. Grand canonical simulations of electrochemical interfaces in implicit solvation models. The Journal of chemical physics, 150(4):041730, 2019.

[44] Yufeng Huang, Robert J Nielsen, and William A Goddard III. Reaction mechanism for the hydrogen evolution reaction on the basal plane sulfur vacancy site of mos2 using grand canonical potential kinetics. Journal of the American Chemical Society, 140(48):16773-16782, 2018. 